\title{
Temperature Correlators of the Impenetrable Bose Gas as an Integrable System
}

\author{
A. R. Its ${ }^{1}$, A. G. Izergin ${ }^{2, \star}$, and V. E. Korepin ${ }^{3}$ \\ ${ }^{1}$ Leningrad University, Leningrad, USSR \\ ${ }^{2}$ Leningrad Branch of the V. A. Steklov Mathematical Institute, Leningrad, USSR, 191011 \\ ${ }^{3}$ ITP, SUNY at Stony Brook, NY 11794-3840, USA
}

\begin{abstract}
It is shown that the temperature equal-time correlators of impenetrable bosons in one space dimension are described by a classical integrable system. Partial differential equations for two-point as well as for multipoint correlators are obtained. The short-distance and low-density expansions are constructed.
\end{abstract}

\section{Introduction}

The Hamiltonian of the one-dimensional non-relativistic Bose gas [1] is

$$
H=\int_{-\infty}^{\infty}\left(\partial_{z} \psi^{+} \partial_{z} \psi+c \psi^{+} \psi^{+} \psi \psi-h \psi^{+} \psi\right) d z
$$

Here $\psi(z), \psi^{+}(z)$ are canonical Bose fields, $\left[\psi(z), \psi^{+}(y)\right]=\delta(z-y)$ and $h$ is a chemical potential. Only the case of impenetrable bosons is considered below, the corresponding value of the coupling constant being $c=+\infty$. The thermodynamics of the model was constructed in paper [2]. At zero temperature the thermal equilibrium state is the ground state of the Hamiltonian, representing a Fermi zone. All the states of particles with momenta $k,-q \leqq k \leqq q$ are filled (here $q=h^{1 / 2}$ is the Fermi momentum). At temperature $T>0$, the thermal equilibrium distribution of particles is given by the Fermi weight

$$
w(k, h, T)=(1+\exp \{\varepsilon(k) / T\})^{-1},
$$

where $\varepsilon(k)=k^{2}-h$ is a particle energy. Gas density $D$ is

$$
D=\frac{1}{2 \pi} \int_{-\infty}^{\infty} w(k, h, T) d k
$$

* Permanent address: Leningrad Branch of the V. A. Steklov Mathematical Institute, Fontanka 27, SU-191011 Leningrad, USSR 
and the kinetical energy density $E$ is given as

$$
E=\frac{1}{2 \pi} \int_{-\infty}^{\infty} k^{2} w(k, h, T) d k
$$

Chemical potential $h$ determines gas density as a monotone increasing function of $h$. At $T=0, D=0$ at $h=0$, and $0<D<+\infty$ as $0<h<+\infty$. At $T>0, D=0$ at $h=-\infty$ and $0<D<+\infty$ as $-\infty<h<+\infty$.

In this paper equal-time correlators are considered which are mean values of field products with respect to the state of the thermal equilibrium. At $T>0$ the mean value of some operator $O$ is defined as usual

$$
\langle O\rangle_{T}=\operatorname{Tr}(O \exp \{-H / T\}) /(\operatorname{Tr}(\exp \{-H / T\}) .
$$

In particular, the two-point field correlator

$$
\left\langle\psi^{+}(z) \psi(-z)\right\rangle_{T}
$$

(where distance $z$ is a real variable) is a real-valued function of $|z|$. Hence it is sufficient to consider it in the case $z>0$ which is supposed further.

The generating functional for correlators of densities

$$
\langle\exp \{\alpha Q(z)\}\rangle_{T}
$$

will also be considered. Here $Q(z)$ is the operator of the number of particles on an interval of length $z$

$$
Q(z)=\int_{0}^{z} \psi^{+}(y) \psi(y) d y
$$

and $\alpha$ is a free parameter. One easily extracts the two-point density correlator as

$$
\left\langle\psi^{+}(z) \psi(z) \psi^{+}(0) \psi(0)\right\rangle_{T}=\left.(1 / 2) \partial_{z}^{2} \partial_{\alpha}^{2}\langle\exp \{\alpha Q(z)\}\rangle_{T}\right|_{\alpha=0} .
$$

The value of the generating functional at $\alpha=-\infty$,

$$
P(z, t, h)=\left.\langle\exp \{\alpha Q(x)\}\rangle_{T}\right|_{\alpha=-\infty},
$$

has also a clear physical sense giving a probability that there are no particles of the gas on interval $[0, z]$ in the state of the thermal equilibrium. Function $P$ will be called "the emptiness formation probability."

Multi-point equal-time correlators

$$
\left\langle\psi^{+}\left(z_{1}^{+}\right) \ldots \psi^{+}\left(z_{N}^{+}\right) \psi\left(z_{1}^{-}\right) \ldots \psi\left(z_{N}^{-}\right)\right\rangle_{T}
$$

will also be considered.

The correlators described above are considered in this paper. Our approach is naturally related to the approach of papers [3-5]. The further contents of the paper is as follows. In Sects. 2-6 the simplest correlators (1.6), (1.7), (1.10) are considered. In Sect. 2 it is demonstrated that all of them are generated by the same linear integral operator. Completely integrable partial differential equations for these correlators representing the main results of this paper are given in Sect. 3. The derivation of these results is explained in Sect. 4 where the completely integrable dynamical system describing temperature correlators is 
constructed. The small distance behaviour of correlators is considered in Sect. 5 and the low density expansion is given in Sect. 6. Multi-point correlators are calculated in Sect. 7.

\section{Correlation Functions and Fredholm Operators}

Only the simplest two-point temperature correlators (1.6), (1.7), (1.10) are considered up to Sect. 7, where multi-point correlators are dealt with. These simplest correlators are functions of three variables $z, h, T$. The essential dependence is, however, on two parameters only [3], namely, on normalized distance $x$ and chemical potential $t$,

$$
x=z T^{1 / 2}, t=h / T .
$$

One has for the field correlator (1.6),

$$
\left\langle\psi^{+}(z) \psi(-z)\right\rangle_{T}=T^{1 / 2} g(x, t),
$$

and correlators (1.7), (1.10) at $x, t$ fixed do not depend on temperature explicitly.

All the correlators mentioned can be expressed in terms of the linear integral operator $\mathscr{K}$ acting on any function of spectral parameter $\lambda$ as follows:

$$
(\mathscr{K} f)(\lambda)=\int_{-\infty}^{\infty} K(\lambda, \mu) f(\mu) d \mu
$$

Kernel $K(\lambda, \mu)$ is

$$
K(\lambda, \mu)=\sqrt{\vartheta(\lambda)} \frac{\sin x(\lambda-\mu)}{(\lambda-\mu)} \sqrt{\vartheta(\mu)} .
$$

Fermi weight $\vartheta(\lambda),(1.2),(2.1)$ is given as

$$
\vartheta(\lambda)=w(\lambda, t, 1)=\left(1+\exp \left\{\lambda^{2}-t\right\}\right)^{-1} .
$$

$[\vartheta(\lambda)$ is, of course, also a function of $t$; this dependence will not be written down explicitly.] It is convenient to describe properties of operator $\mathscr{K}$ in terms of functions $f_{+}(\lambda, x, t, \gamma)$ and $f_{-}(\lambda, x, t, \gamma)$, the dependence on $x, t, \gamma$ being also as a. rule suppressed in notations: $f_{ \pm}(\lambda) \equiv f_{ \pm}(\lambda, x, t, \gamma)$. These functions are defined as the solutions of linear integral equations

$$
f_{ \pm}(\lambda)-\gamma \int_{-\infty}^{\infty} K(\lambda, \mu) f_{ \pm}(\mu) d \mu=e_{ \pm}(\lambda)
$$

Here $\gamma$ is a real parameter and functions $e_{ \pm}(\lambda)$ are

$$
e_{ \pm}(\lambda)=\sqrt{\vartheta(\lambda)} \exp \{ \pm i \lambda x\}
$$

Of importance will be the $2 \times 2$ matrix of "potentials" $B_{l m}(x, t, \gamma)(l=+,-$; $m=+,-)$

$$
B_{l m}(x, t, \gamma)=\gamma \int_{-\infty}^{\infty} e_{l}(\lambda) f_{m}(\lambda) d \lambda
$$


It is a real symmetrical matrix with two independent matrix elements $B_{++}, B_{+-}$,

$$
B_{++}^{*}=B_{++}=B_{--} ; B_{+-}^{*}=B_{+-}=B_{-+} .
$$

Now all the notations necessary to express the correlators in terms of operator $\mathscr{K}$ are introduced.

Field correlator (1.6), (2.2) is represented as

$$
g(x, t)=\left.(1 / 4) B_{++}(x, t, \gamma) \Delta(x, t, \gamma)\right|_{\gamma=2 / \pi},
$$

where $\Delta$ is a Fredholm determinant

$$
\Delta(x, t, \gamma)=\operatorname{det}(1-\gamma \mathscr{K}) .
$$

At the end of this section it will be shown that representation (2.10) can be obtained from the well-known Lenard formula [3] by means of the Fourier transform.

The expression for correlator (1.7) is still simpler [6],

$$
\langle\exp \{\alpha Q(z)\}\rangle_{T}=\left.\Delta(x / 2, t, \gamma)\right|_{\gamma=(1-\exp \{\alpha\}) / \pi} .
$$

The critical value $\gamma=1 / \pi(\alpha=-\infty)$ corresponds to the emptiness formation probability (1.10)

$$
P(z, h, T)=\left.\Delta(x / 2, t, \gamma)\right|_{\gamma=1 / \pi} .
$$

So correlators are represented in terms of operator $\mathscr{K}$.

Let us now derive representation (2.10). The result of paper [3] is

$$
g(x, t)=\left.(\gamma / 2) \varrho(x,-x) \operatorname{det}\left(1-\gamma \theta_{T}\right)\right|_{\gamma=2 / \pi} .
$$

Here $\theta_{T}$ is a linear integral operator with a difference kernel acting on interval $[-x, x]$

$$
\left(\theta_{T} \varphi\right)(\xi)=\int_{-x}^{x} \theta_{T}(\xi-\eta) \varphi(\eta) d \eta,
$$

the kernel $\theta_{T}(\xi-\eta)$ being a Fourier transform of Fermi weight (2.5),

$$
\theta_{T}(\xi-\eta)=\frac{1}{2} \int_{-\infty}^{\infty} \exp \{i(\xi-\lambda) \lambda\} \vartheta(\lambda) d \lambda .
$$

Factor $\varrho(x,-x)$ is the special value of the resolvent $\varrho(\xi, \eta)$,

$$
\varrho(\xi, \eta)-\gamma \int_{-x}^{x} \theta_{T}\left(\xi-\xi^{\prime}\right) \varrho\left(\xi^{\prime}, \eta\right) d \xi^{\prime}=\theta_{T}(\xi-\eta) .
$$

Let us show now the equivalence of (2.14) and (2.10). Consider the integral equation

$$
\varphi(\xi)-\gamma \int_{-x}^{x} \theta_{T}\left(\xi-\xi^{\prime}\right) \varphi\left(\xi^{\prime}\right) d \xi^{\prime}=\Phi(\xi) .
$$

Going to Fourier transform

$$
\varphi(\xi)=\int_{-\infty}^{\infty} \exp \{-i \lambda \xi\} \sqrt{\vartheta(\lambda)} f(\lambda) d \lambda,
$$


one has the equation for $f(\lambda)$

$$
\begin{gathered}
f(\lambda)-\gamma \int_{-\infty}^{\infty} K(\lambda, \mu) f(\mu) d \mu=F(\lambda), \\
F(\lambda)=\frac{1}{2 \pi \sqrt{\vartheta(\lambda)}} \int_{-\infty}^{\infty} d \xi \exp \{i \lambda \xi\} \Phi(\xi) .
\end{gathered}
$$

Kernel $K(\lambda, \mu)$ here is exactly kernel (2.4). It follows then that the Fredholm determinants of operators (2.3) and (2.15) are the same,

$$
\operatorname{det}(1-\gamma \mathscr{K})=\operatorname{det}\left(1-\gamma \theta_{T}\right)=\Delta(x, t, \gamma) .
$$

Making a Fourier transform in Eq. (2.17) one obtains that

$$
\varrho(x,-x)=(2 \gamma)^{-1} B_{++}
$$

which completes the proof of (2.10).

\section{Partial Differential Equations for Temperature Correlation Functions}

Here main results (obtained further in Sects. 4-6) concerning the simplest equaltime two-point correlators are summarized.

Recall that correlators (1.6), (1.7), (1.10) are expressed (2.2), (2.10), (2.12), (2.13) in terms of Fredholm determinant $\Delta(x, t, \gamma)(2.11)$ and function $B_{++}(x, t, \gamma)(2.8)$ at special values of parameter $\gamma$. It is shown in the next section that at any $\gamma$

$$
B_{++}^{2}=-\partial_{x}^{2}(\ln \Delta) \text {, }
$$

and differential equations for $B_{++}$and $\Delta$ are obtained.

The partial differential equation in $x, t$ for function $B_{++}$is

$$
\partial_{t}\left(B_{++}^{2}\right)=1+(1 / 2) \partial_{x}\left(\left(\partial_{x} \partial_{t} B_{++}\right) / B_{++}\right)
$$

Initial conditions are given by the following asymptotics at $x \rightarrow 0$ and $t$ fixed (see Sect. 5)

$$
\begin{aligned}
B_{++}(x, t, \gamma) & =\gamma d(t)+(\gamma d(t))^{2} x+O\left(x^{2}\right), \\
d(t) & =\int_{-\infty}^{\infty} \vartheta(\lambda) d \lambda,
\end{aligned}
$$

and by the requirement that

$$
\lim _{t \rightarrow-\infty} B_{++}(x, t, \gamma)=0 \text {. }
$$

As shown in Sect. $6, B_{++}$decreases at $t \rightarrow-\infty$ and can be expanded into a Taylor series in $\exp \{t\}$ at $t \rightarrow-\infty$ and $x$ fixed.

The Eq. (3.2) can be obtained from the sine-Gordon equation by means of deformations proposed in paper [7]. 
Fredholm determinant $\Delta$ and hence correlators (1.7), (1.10) also satisfy the partial differential equation valid for any $\gamma$. Putting

$$
\sigma(x, t, \gamma)=\ln \Delta(x, t, \gamma) \text {, }
$$

one has (see Sect. 4)

$$
\left(\partial_{t} \partial_{x}^{2} \sigma\right)^{2}=-4\left(\partial_{x}^{2} \sigma\right)\left[2 x \partial_{t} \partial_{x} \sigma+\left(\partial_{t} \partial_{x} \sigma\right)^{2}-2 \partial_{t} \sigma\right]
$$

Initial conditions are [see (3.3)]

$$
\begin{gathered}
\sigma=-\gamma d(t) x-\gamma^{2} d^{2}(t) x^{2} / 2+O\left(x^{3}\right), \\
\lim _{t \rightarrow-\infty} \sigma(x, t, \gamma)=0 .
\end{gathered}
$$

At $t \rightarrow-\infty$ (and $x$ fixed) $\sigma$ is a decreasing function of $t$ and can be expanded into a Taylor series in $\exp \{t\}$.

Differential equations for $B_{++}$and $\sigma$ allow us to obtain asymptotics of correlators in various regimes of parameters. The short distance asymptotics $(x \rightarrow 0, t-$ fixed $)$ is given in Sect. 5 , where the complete asymptotical expansion is constructed. The limit $t \rightarrow-\infty$ corresponds to $D \rightarrow 0$. The complete low density expansion of correlators is given in Sect. 6. The most subtle question is about the long distance asymptotics $(x \rightarrow \infty)$. The solution of this problem requires applying the formalism of classical inverse scattering method. The results will be given in a separate paper.

To conclude let us discuss the zero temperature limit $T=0$. In this limit the integral operator $\mathscr{K}(2.3)$ is reduced to the integral operator $\mathscr{K}_{0}$ acting on interval $[-q, q]\left(q \equiv h^{1 / 2}\right)$ with the kernel

$$
K_{0}(\lambda, \mu)=(\lambda-\mu)^{-1} \sin x(\lambda-\mu) .
$$

Operator $\theta_{T}(2.15)$ becomes the operator $\theta_{0}$ acting on interval $[-x, x]$ with the kernel

$$
\theta_{0}(\xi-\eta)=(\xi-\eta)^{-1} \sin q(\xi-\eta)
$$

Fredholm determinant $\Delta$ at $T=0$ depends only on a product $\tau$ of $x$ and $t^{1 / 2}$,

$$
\left.\Delta(x, t, \gamma)\right|_{T=0}=\Delta_{0}(\tau, \gamma) ; \tau=x t^{1 / 2}=z h^{1 / 2} .
$$

Equation (3.6) rewritten for function

$$
\sigma_{0}(\tau)=\tau\left(\partial \ln \Delta_{0}(\tau, \gamma) / \partial \tau\right)
$$

becomes (prime denotes $\partial / \partial \tau$ )

$$
\left(\tau \sigma_{0}^{\prime \prime}\right)^{2}=-4\left(\tau \sigma_{0}^{\prime}-\sigma_{0}\right)\left(4 \tau \sigma_{0}^{\prime}+\left(\sigma_{0}^{\prime}\right)^{2}-4 \sigma_{0}\right)
$$

reproducing the famous result of paper [4].

\section{Correlators as a Completely Integrable System}

To derive Eqs. (3.2), (3.6) for correlators one begins with integral equations (2.6) for functions $f_{ \pm}(\lambda)$

$$
f_{ \pm}(\lambda)-\gamma \int_{-\infty}^{\infty} K(\lambda, \mu) f_{ \pm}(\mu) d \mu=e_{ \pm}(\lambda)
$$


Here

$$
e_{ \pm}(\lambda)=\sqrt{\vartheta(\lambda)} \exp \{ \pm i \lambda x\}
$$

and kernel $K(\lambda, \mu)(2.4)$ can be represented as

$$
\begin{aligned}
& K(\lambda, \mu)=[2 i(\lambda-\mu)]^{-1}\left[e_{+}(\lambda) e_{-}(\mu)-_{-}(\lambda) e_{+}(\mu)\right], \\
& K(\lambda, \mu)=K(\mu, \lambda) .
\end{aligned}
$$

It should be noted that the resolvent kernel $R(\lambda, \mu)$ defined as

$$
R(\lambda, \mu)-\gamma \int_{-\infty}^{\infty} K\left(\lambda, \lambda^{\prime}\right) R\left(\lambda^{\prime}, \mu\right) d \lambda^{\prime}=K(\lambda, \mu)
$$

[which can be rewritten for operators as $(1-\gamma \mathscr{K}) \mathscr{R}=\mathscr{K}]$ may be represented in a form similar to (4.3) which appears to be extremely useful,

$$
R(\lambda, \mu)=[2 i(\lambda-\mu)]^{-1}\left[f_{+}(\lambda) f_{-}(\mu)-f_{-}(\lambda) f_{+}(\mu)\right] .
$$

To prove this one multiplies both sides of Eq. (4.4) by $(\lambda-\mu)$, representing the factor $(\lambda-\mu)$ under the integral in the second term as $(\lambda-\mu)=\left(\lambda-\lambda^{\prime}\right)+\left(\lambda^{\prime}-\mu\right)$ and then using Eqs. (4.1) (as well as equations conjugated to them).

Now turn to the derivation of Eqs. (3.2) and (3.6). The main idea is to consider (4.1) as a Gel'fand-Levitan-Marchenko-type equation for some integrable system, the function $\vartheta(\lambda)$ playing the role of a reflection coefficient. Though $\vartheta(\lambda)$ for impenetrable bosons is fixed $(2.5)$ as $\vartheta(\lambda)=\left(1+\exp \left\{\lambda^{2}-t\right\}\right)^{-1}$, all the considerations in this section are valid also if $\vartheta$ is an arbitrary function of the difference $\left(\lambda^{2}-t\right)$ decreasing as $\left(\lambda^{2}-t\right) \rightarrow+\infty$, i.e. satisfying requirements

$$
\left(2 \lambda \partial_{t}+\partial_{\lambda}\right) \vartheta(\lambda)=0,\left.\vartheta\right|_{\left(\lambda^{2}-t\right) \rightarrow+\infty}=0 .
$$

The dependence of solutions of partial differential equations (3.2), (3.6) on arbitrary function $\vartheta(\lambda)$ enters in initial values (3.3), (3.7) containing function $d(t)$ $=\int \vartheta(\lambda) d \lambda$. It can easily be shown that for a given $d(t)$ function $\vartheta,(4.6)$ is defined uniquely (and vice versa).

It is worth mentioning that the idea of obtaining differential equations for solutions of integral equations is not quite new; in different aspects it was used in papers [8-11].

Let us derive now the partial differential equation (3.2) for function $B_{++}(x, t, \gamma)$. The following relations can be obtained from integral equations (4.1):

$$
\begin{aligned}
\partial_{x} F(\lambda) & =\left(i \lambda \sigma_{3}+Q\right) F(\lambda), \\
\left(2 \lambda \partial_{t}+\partial_{\lambda}\right) F(\lambda) & =\left(i x \sigma_{3}-i \partial_{t} V\right) F(\lambda) .
\end{aligned}
$$

Here $F(\lambda)$ is a two-component vector function

$$
F(\lambda)=\left(\begin{array}{l}
f_{+}(\lambda) \\
f_{-}(\lambda)
\end{array}\right)
$$

and $2 \times 2$ matrices $\sigma_{3}, Q, V$ are

$$
\sigma_{3}=\left(\begin{array}{rr}
1 & 0 \\
0 & -1
\end{array}\right) ; \quad Q=\left(\begin{array}{cc}
0 & B_{++} \\
B_{++} & 0
\end{array}\right) ; \quad V=\left(\begin{array}{ll}
B_{+-} & -B_{++} \\
B_{++} & -B_{+-}
\end{array}\right) .
$$


"Potentials" $B_{l m}(x, t, \gamma)(l=+,-; m=+,-)$ were introduced in (2.8), (2.9); it is to be stressed that they do not depend on spectral parameter $\lambda$.

To prove (4.7) one first differentiates Eqs. (4.1) with respect to $x$ thus obtaining

$$
\left[(1-\gamma \mathscr{K}) \partial_{x} f_{ \pm}\right](\lambda)-\gamma\left[\left(\partial_{x} \mathscr{K}\right) f_{ \pm}\right](\lambda)= \pm i \lambda e_{ \pm}(\lambda)
$$

Then operator $(1-\gamma \mathscr{K})^{-1}$ should be applied to both sides. Noting that the kernel of operator $\partial_{x} \mathscr{K}$ [see (4.2)] is proportional to the sum of two onedimensional projectors

$$
2 \partial_{x} K(\lambda, \mu)=e_{+}(\lambda) e_{-}(\mu)+e_{-}(\lambda) e_{+}(\mu)
$$

one easily calculates the action of operator $(1-\gamma \mathscr{K})^{-1}$ on the left-hand side. To calculate the action of this operator on the right-hand side one uses relation $(1-\gamma \mathscr{K})(1+\gamma \mathscr{R})=1$ and formula (4.5) for the resolvent kernel.

The proof of (4.8) is based essentially on properties (4.6) of function $\vartheta(\lambda)$. It is more cumbersome (see Appendix A).

Relations (4.7), (4.8) are valid for any $\lambda$ and can be regarded as a zero curvature representation (similar to those constructed in [7]) for some nonlinear evolutionary partial differential equations for potentials $B$. The compatibility condition

$$
\left[\partial_{x}-i \lambda \sigma_{3}-Q, 2 \lambda \partial_{t}+\partial_{\lambda}-i x \sigma_{3}+i \partial_{t} V\right]=0
$$

leads to the following system of equations for functions $B_{++}, B_{+-}$,

$$
\begin{aligned}
\partial_{t} \partial_{x} B_{+-} & =\partial_{t}\left(B_{++}^{2}\right), \\
\partial_{t} B_{+-} & =x+(1 / 2)\left(\partial_{x} \partial_{t} B_{++} / B_{++}\right) .
\end{aligned}
$$

Using the fact that $B_{l m} \rightarrow 0$ at $t \rightarrow-\infty$, one can transform (4.13) into

$$
\partial_{x} B_{+-}=B_{++}^{2}
$$

[it is worth mentioning that this simple equation can also be derived directly from definition (2.8) of potentials $B$ using only Eq. (4.7) for $\partial_{x} F$ ].

Eliminating now function $B_{+-}$from the system (4.14), (4.15) one comes just to Eq. (3.2) for potential $B_{++}$which is therefore established.

Let us explain now how the partial differential equation (3.6) for $\sigma=\ln \Delta$ is derived. To this and let us first calculate the differential $d \sigma=\left(\partial_{x} \sigma\right) d x+\left(\partial_{t} \sigma\right) d t$. Partial derivatives turn out to be

$$
\begin{aligned}
& \partial_{x} \sigma=-B_{+-} \\
& \partial_{t} \sigma=-x \partial_{t} B_{+-}+(1 / 2)\left(\partial_{t} B_{+-}\right)^{2}-(1 / 2)\left(\partial_{t} B_{++}\right)^{2} .
\end{aligned}
$$

To obtain the $x$ derivative is rather simple,

$$
\partial_{x} \sigma=\partial_{x} \ln \operatorname{det}(1-\gamma \mathscr{K})=-\gamma \operatorname{tr}\left[(1-\gamma \mathscr{K})^{-1} \partial_{x} \mathscr{K}\right],
$$

which by means of (4.12) is reduced to (4.16). Equation for $\partial_{t} \sigma$ is obtained not so straightforwardly, the derivation being given in Appendix A. 
To obtain Eq. (3.6) it is now sufficient to substitute $B_{+-}=-\partial_{x} \sigma$ and $B_{++}$ $=\left(-\partial_{x}^{2} \sigma\right)^{1 / 2}$ into (4.17).

It is interesting to note that the equality of mixed derivatives $\partial_{x} \partial_{t} \sigma=\partial_{t} \partial_{x} \sigma$, plus Eq. (4.15) lead to Eq. (3.2) for $B_{++}$.

\section{Short Distance Asymptotics}

Here the behaviour of correlators at small $x$ is analyzed. We study potentials $B_{++}, B_{+-}$as well as functions $\sigma=\ln \Delta$ and $g=B_{++} \Delta / 4$. At small $x$ the kernel $K$ of the integral operator $\mathscr{K}(2.1)$ is small and therefore a perturbation theory in $\mathscr{K}$ is valid. It is easily seen from (2.6) and (2.8) that potentials $B$ can be represented as

$$
\begin{aligned}
& B_{+-}=\int_{-\infty}^{\infty} s(\lambda) d \lambda, \\
& B_{++}=\int_{-\infty}^{\infty} \exp \{2 i \lambda x\} s(\lambda) d \lambda,
\end{aligned}
$$

where $s(\lambda)$ satisfies the linear integral equation

$$
s(\lambda)-\gamma \vartheta(\lambda) \int_{-\infty}^{\infty} \frac{1-\exp \{-2 i x(\lambda-\mu)\}}{2 i(\lambda-\mu)} s(\mu) d \mu=\gamma \vartheta(\lambda) .
$$

Expanding $s(\lambda)$ into the Taylor series in $x$ one has

$$
s(\lambda)=\sum_{k=0}^{\infty} s_{k}(\lambda) x^{k}
$$

[where $s_{k}(\lambda)$ are also functions of $t$ and $\gamma$ ]. For $s_{k}$ the recursion relation is readily obtained

$$
\begin{aligned}
s_{m}(\lambda)= & s_{0}(\lambda) \sum_{k=0}^{m-1}(2 i)^{m-k-1}((m-k) !)^{-1} \\
& \times \int_{-\infty}^{\infty}(\mu-\lambda)^{m-k-1} s_{k}(\mu) d \mu ; s_{0}(\lambda) \equiv \gamma \vartheta(\lambda),
\end{aligned}
$$

which allows us to calculate any $s_{m}$ in the explicit but somewhat lengthy form. So the complete short-distance expansions for functions $B_{+-}(5.1), B_{++}(5.2)$ as well as for $\sigma$

$$
\sigma=\ln \operatorname{det}(1-\gamma \mathscr{K})=-\int_{0}^{x} B_{+-}(\xi, t, \gamma) d \xi
$$

and $g$

$$
g(x, t)=(1 / 4) B_{++}(x, t, \gamma=2 / \pi) \exp \{\sigma(x, t, \gamma=2 / \pi)\}
$$

are obtained. Coefficients of these expansions are expressed in terms of functions $\beta_{l}(t, \gamma)$ which are moments of the function $\vartheta(\lambda)$,

$$
\beta_{l}(t, q)=\gamma \int_{-\infty}^{\infty} \lambda^{l} \vartheta(\lambda) d \lambda \quad\left(\beta_{2 k+1}=0\right)
$$

[function $\beta_{0}(t)=\gamma d(t)$, see (3.3)]. 
Let us give first several terms

$$
\begin{gathered}
B_{++}=\beta_{0}+\beta_{0}^{2} x+\left(\beta_{0}^{3}-2 \beta_{2}\right) x^{2} \\
+\left(\beta_{0}^{4}-(4 / 3) \beta_{0} \beta_{2}\right) x^{3}+O\left(x^{4}\right), \\
B_{+-}=\beta_{0}+\beta_{0}^{2} x+\beta_{0}^{3} x^{2}+\left(\beta_{0}^{4}-(4 / 3) \beta_{0} \beta_{2}\right) x^{3} \\
+\left(\beta_{0}^{5}-(5 / 3) \beta_{0}^{2} \beta_{2}\right) x^{4}+O\left(x^{5}\right), \\
\sigma=-\beta_{0} x-\beta_{0}^{2} x^{2} / 2-\beta_{0}^{3} x^{3} / 3-\left[\left(\beta_{0}^{4} / 4\right)-\left(\beta_{0} \beta_{2} / 3\right)\right] x^{4} \\
-\left[\left(\beta_{0}^{5} / 5\right)-\left(\beta_{0}^{2} \beta_{2} / 3\right] x^{5}+O\left(x^{6}\right) .\right.
\end{gathered}
$$

From these expansions one easily obtains the expansion for the function $g(x, t)$ which defines (2.2) the two-point correlator; the answer is

$$
\langle\psi(z) \psi(-z)\rangle_{T}=D\left[1-2(E / D) z^{2}+(8 / 3) E z^{3}\right]+O\left(z^{4}\right),
$$

where $D$ is density (1.3) and $E$ is kinetical energy density (1.4).

The first two terms in (5.9), (5.11) give initial conditions (3.3), (3.7) for partial differential equations (3.2), (3.6). These equations allow, of course, to reproduce the same small $x$ expansion (see Appendix B).

\section{Low Density Expansion}

Turn now to the expansion of correlators at low densities. It is easy to see that gas density $D(1.3)$ is small at large negative $t(D \rightarrow 0$ at $t \rightarrow-\infty)$. For convenience the variable $\zeta$ is introduced here,

$$
\zeta=-\exp \{t\} ; \zeta \rightarrow 0 \text { at } t \rightarrow-\infty .
$$

It is shown below that functions $B_{+_{+}}, B_{+-}, \sigma=\ln \Delta$, and $g(2.2)$ can be expanded into absolutely convergent Taylor series in $\zeta$ at $\zeta \rightarrow 0$ ( $x$ fixed)

$$
\begin{aligned}
B_{++} & =\sum_{k=1}^{\infty} b_{k} \zeta^{k}, \\
B_{+-} & =\sum_{k=1}^{\infty} c_{k} \zeta^{k}, \\
\sigma & =\sum_{k=1}^{\infty} \sigma_{k} \zeta^{k}=-\int_{0}^{x} B_{+-}(y, t) d y, \\
g & =\sum_{k=1}^{\infty} g_{k} \zeta^{k}=\frac{1}{4} B_{++}(x, t, 2 / \pi) \exp \{\sigma(x, t, 2 / \pi)\} .
\end{aligned}
$$

Quantities $b_{k}, c_{k}, \sigma_{k}$ here are functions of $x$ and $\gamma$ and $g_{k}$ are functions of $x$.

Explicit formulae for coefficients of these series as well as the lower bound for the convergence radius are given below. Our starting point is again representations (5.1), (5.2) for $B$ and the integral equation (5.3). Putting into (5.3) function $s(\lambda)$ in the form

$$
s(\lambda)=\sum_{k=1}^{\infty} \zeta^{k} S_{k}
$$


$\left(s_{k}\right.$ are functions of $x$ and $\left.\gamma\right)$ and representing $\vartheta(\lambda)(2.5)$ as

$$
\vartheta(\lambda)=-\sum_{k=1}^{\infty} \zeta^{k} \exp \left\{-k \lambda^{2}\right\},
$$

one obtains recursion relations for $s_{k}$ which can be solved in the explicit form. So one derives for coefficients $b_{k}$ and $c_{k}$ in (6.2),

$$
\begin{aligned}
b_{k}= & \sum_{l=1}^{k} b_{k}^{(l)} ; b_{k}^{(1)}=-\gamma(\pi / k)^{1 / 2} \exp \left\{-x^{2} / k\right\}, \\
b_{k}^{(l)}= & \left(-\gamma \pi^{1 / 2}\right)^{l}(k-l+1)^{-1 / 2} \exp \left\{-x^{2} / k\right\} \\
& \times \int_{0}^{x} d x_{1} \ldots d x_{l-1} \exp \left\{-\frac{k}{(l-1)(k-l+1)}\left(x_{l-1}-\frac{l-1}{k} x\right)^{2}\right\} \\
& \times \exp \left\{-\sum_{m=1}^{l-2} \frac{m+1}{m}\left(x_{m}-\frac{m}{m+1} x_{m+1}\right)^{2}\right\},
\end{aligned}
$$

and

$$
\begin{aligned}
c_{k}= & \sum_{l=1}^{k} c_{k}^{(l)} ; c_{k}^{(1)}=-\gamma(\pi / k)^{1 / 2} \\
c_{k}^{(l)}= & \left(-\gamma \pi^{1 / 2}\right)^{l}(k-l+1)^{-1 / 2} \\
& \times \int_{0}^{x} d x_{1} \ldots d x_{l-1} \exp \left\{-\frac{k}{(l-1)(k-l+1)} x_{k-1}^{2}\right\} \\
& \times \exp \left\{-\sum_{m=1}^{l-2} \frac{m+1}{m}\left(x_{m}-\frac{m}{m+1} x_{m+1}\right)^{2}\right\} .
\end{aligned}
$$

These formulae allow us to obtain explicit expansions at $t \rightarrow-\infty$ for all the functions in (6.2). They also allow one to obtain the following estimates uniform in $x$ :

$$
\begin{gathered}
\left|b_{k}\right| \leqq A_{k}, \quad\left|c_{k}\right| \leqq A_{k}, \\
A_{k} \equiv \gamma(\pi / k)^{1 / 2} \sum_{l=0}^{k-1}(\pi \gamma)^{l},
\end{gathered}
$$

which guarantees that expansions (6.3) [and also (6.2)] are absolutely convergent in the following regions:

(i) At $|\zeta|<1(t<0)$ if $\gamma \leqq 1 / \pi$.

(ii) At $|\zeta|<(\pi \gamma)^{-1}(t<-\ln (\pi \gamma))$ if $\gamma>1 / \pi$.

Let us give several first terms of expansions for $\sigma$ and $g$

$$
\begin{aligned}
\sigma= & -\gamma \pi^{1 / 2} x \exp \{t\}+\gamma(\pi / 2)^{1 / 2} x \exp \{2 t\} \\
& +\pi \gamma^{2} \exp \{2 t\} \int_{0}^{x} d y \exp \left\{-2 y^{2}\right\}(x-y)+O(\exp \{3 t\}) \\
& g(x, t)=\frac{\exp \left\{-x^{2}\right\}}{2 \pi} \int_{-\infty}^{\infty} \vartheta(\lambda) d \lambda+O(\exp \{2 t\})
\end{aligned}
$$


or

$$
\left\langle\psi^{+}(z) \psi(-z)\right\rangle_{T}=D \exp \left\{-T z^{2}\right\}\left(h \ll-z^{2} T^{2}\right) .
$$

In conclusion let us emphasize once more that we have shown that at $t \rightarrow-\infty$ (and $x$ fixed) functions $B_{++}, B_{+-}$can be expanded into convergent series in entire degrees of $\exp \{t\}$, the same being true, of course, also for functions $\sigma$ and $g$.

\section{Multi-Point Correlators}

Temperature equal-time multipoint correlators are considered here. Introducing normalized distance $x=z \sqrt{T}$ and chemical potential $t=h / T(2.1)$ one has for correlator (1.11)

$$
\left\langle\psi^{+}\left(z_{1}^{+}\right) \ldots \psi^{+}\left(z_{N}^{+}\right) \psi\left(z_{1}^{-}\right) \ldots \psi\left(z_{N}^{-}\right)\right\rangle_{T}=T^{N / 2} G_{N}\left(\left\{x^{+}\right\},\left\{x^{-}\right\}, t\right),
$$

where $G_{N}\left(\left\{x^{+}\right\},\left\{x^{-}\right\}, t\right) \equiv G_{N}\left(x_{1}^{+}, \ldots, x_{N}^{+} ; x_{1}^{-}, \ldots, x_{N}^{-}, t\right)$.

Lenard [3] represented this correlator as a Fredholm minor of linear integral operator $\theta_{T}^{(N)}$,

$$
\left[\theta_{T}^{(N)} \varphi\right](\xi)=\sum_{j=1}^{N} \int_{x_{2 j-1}}^{x_{2 j}} \theta_{T}(\xi-\eta) \varphi(\eta) d \eta,
$$

where $\theta_{T}(\xi-\eta)$ is a Fourier transform of Fermi weight $\vartheta(\lambda)(2.16)$ and $x_{j}$ 's are all $x_{k}^{+}, x_{k}^{-}$in (7.1) taken in the increasing order

$$
x_{1} \leqq x_{2} \leqq x_{3} \leqq \ldots \leqq x_{2 N} ;\{x\}=\left\{x^{+}\right\} \cup\left\{x^{-}\right\}
$$

Similarly to the two-point case considered in Sect. 2 one can represent the multipoint correlator in terms of another linear integral operator

$$
(\mathscr{K} f)(\lambda)=\int_{-\infty}^{\infty} K(\lambda, \mu) f(\mu) d \mu .
$$

Kernel $K(\lambda, \mu)$ is now

$$
K(\lambda, \mu)=[2 i(\lambda-\mu)]^{-1} \sum_{m=1}^{2 N}(-1)^{m} e_{m}^{+}(\lambda) e_{m}^{-}(\mu),
$$

where functions $e_{m}^{ \pm}$are

$$
e_{m}^{ \pm}(\lambda)=\sqrt{\vartheta(\lambda)} \exp \left\{ \pm i \lambda x_{m}\right\}
$$

It is convenient to introduce functions $f_{m}^{ \pm}(\lambda)$ analogous to $f_{ \pm}(2.6)$,

$$
\begin{aligned}
& f_{m}^{+}(\lambda)-\gamma \int_{-\infty}^{\infty} K(\lambda, \mu) f_{m}^{+}(\mu) d \mu=e_{m}^{+}(\lambda), \\
& f_{m}^{-}(\lambda)-\gamma \int_{-\infty}^{\infty} f_{m}^{-}(\mu) K(\mu, \lambda)=e_{m}^{-}(\lambda)
\end{aligned}
$$

$(m=1, \ldots, 2 N)$ 
Distances $x_{m}(7.2)$ and chemical potential $t$ [entering only function $\vartheta(\lambda)$ $\left.=\left(1+\exp \left\{\lambda^{2}-t\right\}\right)^{-1}\right]$ play a role of parameters in these integral equations. One also defines "potentials" $V_{l m}$ similar to (2.8),

$$
\begin{gathered}
V_{l m}(\{x\}, t, \gamma)=(-1)^{l} \gamma \int_{-\infty}^{\infty} e_{l}^{-}(\mu) f_{m}^{+}(\mu) d \mu, \\
V_{l m}^{*}=V_{l m} ; V_{l m}=(-1)^{l+m} V_{m l} \quad(l, m=1, \ldots, 2 N) .
\end{gathered}
$$

One has the following representation for correlator (7.1) in terms of operator $\mathscr{K}:$

$$
\begin{aligned}
G_{N}\left(\left\{x^{+}\right\},\left\{x^{-}\right\}, t\right)= & (1 / 4)^{N}(-1)^{[(N+1) / 2]} \\
& \times \prod_{j<k} \operatorname{sign}\left(x_{k}^{+}-x_{j}^{+}\right) \operatorname{sign}\left(x_{k}^{-}-x_{j}^{-}\right) \\
& \times\left.\operatorname{Det}_{N}\left(V_{l m}(\{x\}, t, \gamma)\right) \Delta(\{x\}, t, \gamma)\right|_{\gamma=2 / \pi} .
\end{aligned}
$$

Here $[(N+1) / 2]=N / 2$ for $N$ even and $[(N+1) / 2]=(N+1) / 2$ for $N$ odd; $\operatorname{Det}_{N}\left(V_{l m}\right)$ is the determinant of the $N \times N$ matrix with matrix elements $V_{l m}(7.7)$ $(1 \leqq l \leqq N ; N+1 \leqq m \leqq 2 N)$, and $\Delta$ is the Fredholm determinant

$$
\Delta(\{x\}, t, \gamma)=\operatorname{det}(1-\gamma \mathscr{K}) .
$$

In what follows we give differential equations for "potentials" $V_{l m}$ and for the function $\sigma=\ln \Delta$. They are obtained similarly to the two-point case considered in Sects. 3 and 4. So, only the results will be given below. It should, however, be mentioned that the following representation for the kernel $R$ of the resolvent $\mathscr{R}((1-\gamma \mathscr{K}) \mathscr{R}=\mathscr{K})$ is essentially used in deriving the results:

$$
R(\lambda, \mu)=[2 i(\lambda-\mu)]^{-1} \sum_{m=1}^{2 N}(-1)^{m} f_{m}^{+}(\lambda) f_{m}^{-}(\mu) .
$$

[Due to the property

$$
\sum_{l=1}^{2 N}(-1)^{l} f_{l}^{+}(\lambda) f_{l}^{-}(\lambda)=0
$$

there is no pole at $\lambda=\mu$ at the kernel.]

To obtain a zero curvature representation for the multidimensional integrable system $\left(x_{1}, \ldots, x_{2 N}\right.$ are co-ordinates and $t$ is a "time") one applies to Eqs. (7.6) differential operators $\partial_{k} \equiv \partial / \partial x_{k}$ and $\left(2 \lambda \partial_{t}+\partial_{\lambda}\right)$, obtaining a set of $L$-operators and the $M$-operator

$$
\begin{aligned}
\partial_{k} f_{m}^{+}(\lambda)= & \delta_{k m}\left\{i \lambda f_{m}^{+}(\lambda)-\frac{1}{2} \sum_{l=1}^{2 N} f_{l}^{+}(\lambda) V_{l m}\right\} \\
& +\frac{1}{2} f_{k}^{+}(\lambda) V_{k m} \quad(k, m=1, \ldots, 2 N), \\
\left(2 \lambda \partial_{t}+\partial_{\lambda}\right) f_{m}^{+}(\lambda)= & i x_{m} f_{m}^{+}(\lambda) \\
& -i \sum_{l=1}^{2 N} f_{l}^{+}(\lambda) \partial_{t} V_{l m} \quad(m=1, \ldots, 2 N) .
\end{aligned}
$$


Demanding now the compatibility of these equations one comes to the system of partial differential equations for potentials. First write down equations without the time derivatives

$$
\begin{gathered}
\partial_{k} V_{l m}=V_{l k} V_{k m} / 2 \quad(k \neq m, l), \\
\left(\partial_{m}+\partial_{j}\right) V_{j m}=-\frac{1}{2} \sum_{l \neq m, j}^{2 N} V_{j l} V_{l m}, \\
\partial_{m} V_{m m}=-\frac{1}{2} \sum_{l \neq m}^{2 N} V_{m l} V_{l m}
\end{gathered}
$$

[at derivation of (7.14) the fact is taken into account that potentials are going to zero as $t \rightarrow-\infty]$. Equations containing the time derivative are

$$
\begin{aligned}
\partial_{t}\left(\partial_{l}-\partial_{m}\right) V_{l m} & +\left(x_{l}-x_{m}\right) V_{l m}+\left(\partial_{t} V_{m m}-\partial_{t} V_{l l}\right) V_{l m} \\
& +\frac{1}{2} \sum_{p \neq l, m}\left(V_{l p} \partial_{t} V_{p m}-V_{p m} \partial_{t} V_{l p}\right)=0 .
\end{aligned}
$$

One can also easily get the following simple sequences:

$$
\left(\sum_{l=1}^{2 N} \partial_{l}\right) V_{j m}=0 ; \sum_{m=1}^{2 N} V_{m m}=0
$$

So equations for potentials (7.14)-(7.18) are written down.

To derive the equation for function $\sigma$

$$
\sigma(\{x\}, t, \gamma)=\ln \Delta(\{x\}, t, \gamma)=\ln \operatorname{det}(1-\gamma \mathscr{K}),
$$

one first calculates the differential $d \sigma$ with respect to variables $x, t$. The result is

$$
\begin{aligned}
& \partial_{m} \sigma=-V_{m m} / 2, \\
& \partial_{t} \sigma=-\frac{1}{2} \sum_{m=1}^{2 N} x_{m} \partial_{t} V_{m m}+\frac{1}{4} \sum_{m, l=1}^{2 N}\left(\partial_{t} V_{l m}\right)\left(\partial_{t} V_{m l}\right),
\end{aligned}
$$

which by virtue of (7.7), (7.14) gives the equation for $\sigma$,

$$
\begin{aligned}
\partial_{t} \sigma= & \sum_{m=1}^{2 N} x_{m} \partial_{t} \partial_{m} \sigma+\sum_{m=1}^{2 N}\left(\partial_{t} \partial_{m} \sigma\right)^{2} \\
& -\frac{1}{4} \sum_{k \neq m}^{2 N}\left(\partial_{k} \partial_{m} \sigma\right)^{-1}\left(\partial_{t} \partial_{k} \partial_{m} \sigma\right)^{2} .
\end{aligned}
$$

It can be shown also that

$$
\sum_{m=1}^{2 N} \partial_{m} \sigma=0
$$

Considering the equality of mixed derivatives $\partial_{x} \partial_{t} \sigma=\partial_{t} \partial_{x} \sigma$, one obtains from (7.20), (7.21) "conserved currents"

$$
\partial_{t}\left(\sum_{m=1}^{2 N}\left(x_{m}-x_{k}\right) V_{m k} V_{k m}\right)=\partial_{k}\left(\sum_{m, l}^{2 N}\left(\partial_{t} V_{l m}\right)\left(\partial_{t} V_{m l}\right)\right)
$$


It should be noted that the zero-temperature limit of Eqs. (7.14)-(7.20) reproduces (after appropriate change of notations) the corresponding equations of paper [4].

To conclude let us discuss the zero temperature limit of Eq. (7.22) for $\sigma$. In this limit function $\sigma$ depends on $2 N$ variables $\tau_{j}, \tau_{j}=x_{j} t^{1 / 2}=z_{j} h^{1 / 2}$. Recalculating derivatives one comes to be following equation for $\sigma$ at $T=0$ :

$$
\begin{aligned}
& \sum_{i, m} \tau_{i} \tau_{m} \partial_{i} \partial_{m} \sigma+\frac{1}{2} \sum_{m}\left(\partial_{m} \sigma+\sum_{i} \tau_{i} \partial_{i} \partial_{m} \sigma\right)^{2} \\
& \quad=\frac{1}{2} \sum_{k \neq m}\left(\partial_{m} \partial_{k} \sigma\right)^{-1}\left[\partial_{m} \partial_{k} \sigma+\frac{1}{2} \sum_{i} \tau_{i} \partial_{i} \partial_{k} \partial_{m} \sigma\right]^{2}
\end{aligned}
$$
Here $\partial_{i} \equiv \partial / \partial \tau_{i}$. Also, of course, $\sum_{i} \partial_{i} \sigma=0$. Equation (7.25) is a new result even at
zero temperature.

\section{Conclusion}

The impenetrable Bose gas is the simplest non-trivial quantum integrable system which can be obtained by quantization of a classical non-linear system, namely, of the non-linear Schrödinger equation. It is remarkable that after quantization correlation functions are described by a new classical integrable system. The essential point is the possibility to represent correlators in terms of the linear integral operator, the Fredholm determinant playing a role of $\tau$-function of the new integrable system. As representations of the correlators of this kind are known also for other quantum models [6] we hope that the scheme described above is rather general.

It should be noted that the classical integrable system obtained gives the complete description of correlators. The small distance and low density expansions for them was given above. To obtain the asymptotics at large distances is a more difficult problem which requires use of the inverse scattering method. It will be done in the next publication (the first results including the explicit formula for the correlation radius of the two-point field correlator, see in paper $[12])$.

Note added in proofs. I. Gabitov and S. Burtsev informed us that Eq. (3.2) is particular case of Maxwell-Bloch equation

\section{Appendix A}

Here some details of the derivation of $M$ operator (4.8) and of relation (4.17) for $\partial_{t} \sigma$ are given. Considering integral equations (4.1) and differentiating explicitly with respect to $t$, one obtains the following useful formula:

$$
\left[(1-\gamma \mathscr{K}) \partial_{t} f_{ \pm}\right](\lambda)=\left[(1+\gamma \mathscr{K}) \frac{\partial_{t} \vartheta}{2 \vartheta} f_{ \pm}\right](\lambda) .
$$


Taking into account Eqs. (4.4) and (4.5) for the resolvent kernel one derives from (A.1) that

$$
\begin{aligned}
2 \lambda \partial_{t} f_{ \pm}(\lambda)= & 2 \lambda \frac{\partial_{t} \vartheta(\lambda)}{2 \vartheta(\lambda)} f_{ \pm}(\lambda) \\
& +\frac{2 \gamma}{i} \int_{-\infty}^{\infty}\left(f_{+}(\lambda) f_{-}(\mu)-f_{-}(\lambda) f_{+}(\mu)\right) \frac{\partial_{t} \vartheta(\mu)}{2 \vartheta(\mu)} f_{ \pm}(\mu) d \mu \\
& +2 \gamma \int_{-\infty}^{\infty} R(\lambda, \mu) \frac{\mu \partial_{t} \vartheta(\mu)}{\vartheta(\mu)} f_{ \pm}(\mu) d \mu .
\end{aligned}
$$

Differentiating (A.1) with respect to $\lambda$ and performing integration by parts one has

$$
\begin{aligned}
\partial_{\lambda} f_{ \pm}(\lambda)= & \frac{\partial_{\lambda} \vartheta(\lambda)}{2 \vartheta(\lambda)} f_{ \pm}(\lambda) \pm i x f_{ \pm}(\lambda) \\
& +2 \gamma \int_{-\infty}^{\infty} R(\lambda, \mu) \frac{\partial_{\mu} \vartheta(\mu)}{2 \vartheta(\mu)} f_{ \pm}(\mu) d \mu .
\end{aligned}
$$

Summing up Eqs. (A.2) and (A.3) and taking into account that $\left(2 \lambda \partial_{t}+\partial_{\lambda}\right) \vartheta=0$, one comes to

$$
\left(2 \lambda \partial_{t}+\partial_{\lambda}\right) f_{ \pm}(\lambda)= \pm i x f_{ \pm}(\lambda)-i \gamma f_{+}(\lambda) U_{-, \pm}+i \gamma f_{-}(\lambda) U_{+, \pm} .
$$

Here

$$
\begin{gathered}
U_{l m}(x, t, \gamma)=\int_{-\infty}^{\infty} f_{l}(\mu) f_{m}(\mu) \frac{\partial_{t} \vartheta(\mu)}{\vartheta(\mu)} d \mu . \\
(l, m=+,-) .
\end{gathered}
$$

Using again (A.1) one proves after some calculations that

$$
U_{l m}=\partial_{t} \int_{-\infty}^{\infty} e_{l}(\mu) f_{m}(\mu) d \mu,
$$

which allows us to rewrite (A.4) as $M$-operator (4.8). The derivation of relation (4.17) for $\partial_{t} \sigma$ begins with

$$
\partial_{t} \sigma=\partial_{t} \ln \operatorname{det}(1-\gamma \mathscr{K})=-\gamma \operatorname{tr}\left[(1-\gamma \mathscr{K})^{-1} \partial_{t} \mathscr{K}\right]
$$

which by means of (A.1) and (4.5) can be written as

$$
\begin{aligned}
\partial_{t} \sigma & =-\gamma \int_{-\infty}^{\infty} R(\lambda, \lambda) \frac{\partial_{t} \vartheta(\lambda)}{\vartheta(\lambda)} d \lambda \\
& =\frac{i \gamma}{2} \int_{-\infty}^{\infty} d \lambda \frac{\partial_{t} \vartheta(\lambda)}{\vartheta(\lambda)}\left(f_{-}(\lambda) \partial_{\lambda} f_{+}(\lambda)-f_{+}(\lambda) \partial_{\lambda} f_{-}(\lambda)\right)
\end{aligned}
$$

Taking into account Eqs. (A.3) for $\partial_{\lambda} f_{ \pm}$and (A.5), after some calculations one obtains formula (4.17). 


\section{Appendix B}

Explain how the small $x$ expansions (5.9)-(5.13) can be obtained from partial differential equations (3.2), (3.6) with initial conditions (3.3), (3.7) and (3.4), (3.8).

Consider Eq. (3.2) for $\boldsymbol{B}_{++}$in more detail. Representing $\boldsymbol{B}_{++}(x, t)$ (dependence on $\gamma$ will not be written explicitly) as a series in $x$,

$$
\begin{gathered}
B_{++}(x, t)=\sum_{n=0}^{\infty} b_{n}(t) x^{n}, \\
b_{0}(t)=\beta_{0}(t)=\gamma \int_{-\infty}^{\infty} \vartheta(\lambda) d \lambda ; \quad b_{1}(t)=\beta_{0}^{2}(t),
\end{gathered}
$$

one obtains from (3.2) the following recursive system of ordinary differential equations for function $b_{n}(t), n \geqq 2$ :

$$
\begin{aligned}
& \dot{b}_{2}=\frac{1}{2} b_{1} b_{0}^{-1} \dot{b}_{1}+2 b_{0}^{2} \dot{b}_{0}-b_{0}, \\
& \dot{b}_{3}=\frac{1}{3} b_{0}^{-1} b_{2} \dot{b}_{1}+\frac{2}{3} b_{0}^{2} \dot{b}_{1}+2 b_{0} b_{1} \dot{b}_{0}-\frac{2}{3} b_{1}\left(\dot{b} \equiv \partial_{t} b\right)
\end{aligned}
$$

(the general term though rather bulky can also be written explicitly). These equations are easily integrated [initial conditions are $b_{n}(-\infty)=0$ ], the answer being unique. Thus one has for $b_{2}(t)$,

$$
b_{2}(t)=\beta_{0}^{3}(t)-\int_{-\infty}^{t} \beta_{0}(\tau) d \tau .
$$

At first sight this differs from $b_{2}(t)=\beta_{0}^{3}-2 \beta_{2}$ given by expansion (5.9). Nevertheless, they are equal for any function $\vartheta(\lambda)$ satisfying conditions (4.6). To show this it is sufficient to prove that functions $A(t)$ and $B(t)$ defined as

$$
A(t)=\int_{-\infty}^{t} \beta_{0}(\tau) d \tau, \quad B(t)=2 \gamma \int_{-\infty}^{\infty} \lambda^{2} \vartheta(\lambda) d \lambda
$$

are equal. In fact, $A(-\infty)=B(-\infty)=0$ and due to (4.6) also $\partial_{t} A(t)=\partial_{t} B(t)$; so $A(t)=B(t)$.

For $b_{3}$ one obtains from (B.2),

$$
b_{3}(t)=\beta_{0}^{4}-\frac{4}{3} \int_{-\infty}^{t} \beta_{2}(\tau) \dot{\beta}_{0}(\tau) d \tau-\frac{2}{3} \int_{-\infty}^{t} \beta_{0}^{2}(\tau) d \tau,
$$

which, in a similar way, can transform into $b_{3}(t)=\beta_{0}^{4}-(4 / 3) \beta_{2} \beta_{0}$ as given by (5.9). The same is true for the higher terms.

Acknowledgements. We thank M. J. Ablowitz, N. M. Bogolubov, L. D. Faddeev, and A. S. Fokas for discussions. One of us (A.G.I.) would like to thank Professor Abdus Salam, the International Atomic Energy Agency and UNESCO for hospitality at the International Centre for Theoretical Physics, Trieste.

\section{References}

1. Mathematical physics in one dimension. Lieb, E.H., Mattis, D.S. (eds.). New York: Academic Press 1966 
2. Yang, C.N., Yang, C.P.: Thermodynamics of a one-dimensional system of bosons with repulsive delta-function interaction. J. Math. Phys. 10, 1115-1122 (1969)

3. Lenard, A.: One-dimensional impenetrable bosons in thermal equilibrium. J. Math. Phys. 7, 1268-1272 (1965)

4. Jimbo, A., Miwa, T., Mori, Y., Sato, M.: Density matrix of an impenetrable Bose gas and the fifth Painleve transcendent. Physica 1 D, 80-158 (1980)

5. McCoy, B.M., Wu, T.T.: The two-dimensional Ising model. Cambridge, MA: Harvard University Press 1973

6. Korepin, V.E.: Dual field formulation of quantum integrable models. Commun. Math. Phys. 113, 177-190 (1987)

Korepin, V.E.: Generating functional of correlation functions in completely integrable models. Funk Analiz i Ego Prilozh. 23, 15-23 (1989)

7. Burtsev, S.P., Zacharov, V.E., Mikhailov, A.V.: Inverse scattering method with variable spectral parameter. Teor. Mat. Fiz. 70, 323-342 (1987)

8. Krein, M.G.: On integral equations generating differential equations of the second order. Dokl. AN SSSR 97, 21-24 (1954)

9. Krein, M.G.: On definition of potential of a particle in terms of its $S$ function. Dokl. AN SSSR 105, 433-436 (1955)

10. Fokas, A.S., Ablowitz, M.J.: Linearization of the Korteweg-de Vries and Painleve II equations. Phys. Rev. Lett. 47, 1096-1100 (1981)

11. Santini, P.M., Ablowitz, M.J., Fokas, A.S.: The direct linearization of a class of non-linear evolution equations. J. Math. Phys. 25, 2614-2619 (1984)

12. Its, A.R., Izergin, A.G., Korepin, V.E.: Correlation radius for one-dimensional impenetrable bosons. ICTP, preprint IC/89/107

Communicated by A. Jaffe

Received July 11, 1989; in revised form September 28, 1989 